\title{
EU-Kommission kritisiert Zweiteilung des deutschen Gesundheitssystems
}

_ Die EU-Kommission hat am 23. November 2017 eine Analyse der Gesundheitssysteme aller 28 Mitgliedsstaaten vorgestellt. Untersucht wurden die Gesundheit der Bevölkerung, die Risikofaktoren, die Gesundheitsausgaben und die Leistung des Gesundheitssystems im Hinblick auf seine Effektivität, Zugänglichkeit und Anpassungsfähigkeit.

Für Deutschland kommen die Analysten zu einem gemischten Fazit. Zwar habe sich der Gesundheitszustand der Bevölkerung seit dem Jahr 2000 verbessert. Doch die durch Vorsorgemaßnahmen vermeidbare Sterblichkeit liege nur ungefähr im EU-Durchschnitt. Das Gesundheitssystem biete einen großzügigen Leistungskatalog, ein hohes Niveau an Gesundheitsleistungen und einen guten Zugang zur Gesundheitsversorgung. Allerdings spielten die Selbstverwaltungsorgane bei der Gestaltung des Gesundheitssystems eine zu große Rolle und erschwerten mitunter strukturelle
Reformen, die zur Beseitigung von Qualitäts- und Effizienzmängeln erforderlich wären.

Die Analysten erinnern auch daran, dass Deutschland im Jahr 1883 das erste Land der Welt war, das ein landesweites System der gesetzlichen Krankenversicherung einführte. Noch heute erkläre dieses Vermächtnis die beiden wichtigsten Merkmale des Systems: Zum einen die große Zahl an Krankenkassen, zum anderen die starke Rolle der Selbstverwaltungsorgane in der Regulierung. Auch die in der EU unübliche Koexistenz von GKV und PKV habe ihre Wurzeln in ferner Vergangenheit.

\section{MMW-KOMMENTAR}

In der EU-Analyse finden sich weitere scharfe Beobachtungen. So prangern die Experten die hohe Zahl an Krankenhauseinweisungen an, die sie als Zeichen von Lücken in der ambulanten Versorgung ansehen. Hochrelevant erscheint auch die Hinweis, dass höheren Einkommensgruppen der Austritt aus dem GKV-System erlaubt ist. Dies widerspreche dem Solidarprinzip der GKV, nach dem sich die Beiträge nach der Zahlungsfähigkeit richten, die Leistungen aber nach Bedarf bereitgestellt werden. Zudem würden ambulante ärztliche Leistungen für PKV-Patienten höher vergütet als für GKV-Patienten, was zu Bedenken hinsichtlich der Gerechtigkeit führe - v. a. auch hinsichtlich der Wartezeiten. Die Analyse kann also getrost als Steilvorlage für die Pläne der SPD für die Einführung einer Bürgerversicherung in Deutschland angesehen werden.

\section{Hier steht eine Anzeige.}

